\title{
Summing series using residues
}

\section{A. SoFo}

\section{SUMMING SERIES USING RESIDUES}

Differential-difference equations provide a fertile ground for the generation of infinite series. From this point of view the author considers the forced system

$$
\sum_{n=0}^{R}\left(\begin{array}{c}
R \\
R-n
\end{array}\right) c^{k(R-n)} \sum_{r=0}^{n k}\left(\begin{array}{c}
n k \\
r
\end{array}\right) b^{n k-r} f^{(r)}(t-(R-n) a)=w(t)
$$

and generates infinite series of the form

$$
S_{1}(R, k, b, a, t)=\sum_{n=0}^{\infty}\left(\begin{array}{c}
n+R-1 \\
n
\end{array}\right) \frac{b^{n k} e^{-b(t-a n)}(t-a n)^{n k+R k-1}}{(n k+R k-1) !}
$$

which may be represented in closed form, mainly by the use of residue theory. For the most basic case of the arbitrary parameters $(R, k) \equiv(1,1)$ the infinite sum $S_{1}(1,1, b, a, t)$, in different form, has been considered by various mathematicians including Euler, Jensen, Bruwier, Ramanujan and Pölya and Szegö. Their methods of representing $S_{1}(1,1, b, a, t)$ in closed form are different from those developed by the author. The occurrence of $S_{1}(1,1, b, a, t)$ in many guises, is quite prolific in a range of different branches of applications. It appears in the works of Erlang in his study of teletraffic problems, arises in the works of Feller on ruin problems, Hall on coverage processes, Smith on renewal theory, Tijms on stochastic modelling, Woodward and Wake on grazing systems and Keane on the slowing down of neutrons.

$S_{1}(R, k, b, a, t)$ is known as an Abel type series and the author proves that it may be represented in closed form which depends on $k$ dominant zeros of an associated transcendental characteristic function.

In a similar vein the author considers difference-delay systems of the form

$$
\sum_{j=0}^{R}\left(\begin{array}{c}
R \\
R-j
\end{array}\right) c^{k(R-j)} \sum_{r=0}^{j k}\left(\begin{array}{c}
j k \\
r
\end{array}\right)(-b)^{k j-r} f_{n+r-k(R-j) a}=w_{n}
$$

Received 20th May, 1999

Thesis submitted to the Victoria University of Technology, July, 1998. Degree approved, February 1999. Supervisors: Associate Professor P. Cerone and Dr. D. Watson.

Copyright Clearance Centre, Inc. Serial-fee code: 0004-9727/99 \$A2.00+0.00. 
from which infinite sums of the type

$$
S_{2}(R, k, b, a, n)=\sum_{r=0}^{\infty}\left(\begin{array}{c}
r+R-1 \\
r
\end{array}\right)\left(\begin{array}{c}
n-a k r \\
k r+R r-1
\end{array}\right) b^{n-a k r-R k+1},
$$

with arbitrary parameters $(R, k, b, a, n)$, are generated. For the case of the parameters $(R, k) \equiv(1,1)$, Jensen obtained a closed form expression for $S_{2}(1,1, b, a, n)$ and the author proves that $S_{2}(R, k, b, a, n)$ may be expressed in closed form which depends on $k$ dominant zeros of an associated polynomial characteristic function. Moreover, $S_{2}(1, k, b, a, n)$ may be expressed as a generalised hypergeometric function

$$
T_{0(a+1) k} F_{(a+1) k-1}\left[\begin{array}{c}
\frac{k-n-1}{(a+1) k}, \frac{k-n}{(a+1) k}, \ldots, \frac{k-n-1+(a+1) k-1}{(a+1) k} \\
\frac{-n}{a k}, \frac{1-n}{a k}, \ldots, \frac{a k-n-1}{a k}, \frac{2 k-1}{k}, \frac{2 k-2}{k}, \ldots, \frac{k+1}{k}
\end{array} \mid s(a, b, k)\right]
$$

where

$$
T_{0}=\left(\begin{array}{c}
n \\
k-1
\end{array}\right) b^{n-k+1}
$$

and

$$
s(a, b, k)=\left(-\frac{(a+1)^{a+1}}{(a b)^{a}}\right)^{k}
$$

For particular parameter values the author proves that $S_{2}(R, k, b, a, n)$ and its hypergeometric representation reduce to known identities including Kummer's form. Also for particular parameter values $S_{2}(R, k, b, a, n)$ may be certified by the $W Z$ pairs method of Wilf and Zeilberger. The finite form $S_{2 F}(R, k, b, a, n)$ of $S_{2}(R, k, b, a, n)$ may also be associated, for particular parameter values, with the special polynomials of Fibonacri, Jacobsthal, Pell, Fermat and Chebyshev.

Some results of this research may be seen in [1], [2] and [3].

\section{REFERENCES}

[1] A. Sofo, and P. Cerone, 'Generalisation of a waiting time relation', J. Math. Anal. Appl. 214 (1997), 191-206.

[2] A. Sofo and P. Cerone, 'On a Fibonacci related series', Fibonacci Quart. 36 (1998), 211-215.

[3] A. Sofo and P. Cerone, 'Generalisation of Euler's identity', Bull. Austral. Math. Soc. 58 (1998), 359-371.

Faculty of Engineering and Science

Victoria University of 'Technology

PO Box 14428

Melbourne City MC 8001

e-mail: anthony.sofo@vu.edu.au 